See Article page 108.

\section{Commentary: Cellular reprogramming for myocardial regeneration and beyond}

\author{
Akbarshakh Akhmerov, MD, and \\ Danny Ramzy, MD, PhD
}

Ischemic heart disease remains one of the leading causes of death worldwide. The ischemic pathology, most commonly arising from coronary artery disease, results in loss of cardiomyocytes and functional impairment. Although the mammalian heart is capable of regeneration in the embryonic and early postnatal periods, the rate of cardiomyocyte turnover in adult humans is $\leq 1 \%$ per year, which is insufficient for meaningful repair after an ischemic event. ${ }^{1,2}$ Therefore, various regenerative therapies are under investigation for treatment of ischemic heart disease. These encompass exogenous approaches, such as stem/progenitor cell transplantation and infusion of cell-based paracrine factors, and endogenous approaches, including direct in situ conversion of resident non-cardiomyocytes into functional cardiomyocytes. Our knowledge of cellular reprogramming and angiogenic therapies has grown tremendously over the last 20 years because it has needed to, for we as a broader surgical community are peripherally informed at best, and ill informed at worst, on the topic of clinical angiogenic and cellular reprogramming.

In this issue of the Journal, Ryan and colleagues ${ }^{3}$ masterfully inform our surgical community about angiogenic therapy and cellular reprogramming. The authors review a well-described method of direct cellular reprogramming, initially inspired by the observation that fully differentiated fibroblasts can be reprogrammed into pluripotent stem cells

From the Department of Cardiac Surgery, Smidt Heart Institute, Cedars-Sinai Medical Center, Los Angeles, Calif.

Disclosures: Dr Akhmerov's work is funded by the National Heart, Lung, and Blood Institute (Grant T32HL116273-07). Dr Ramzy serves as a speaker for Abiomed and LivaNova.

The Journal policy requires editors and reviewers to disclose conflicts of interest and to decline handling or reviewing manuscripts for which they may have a conflict of interest. The editors and reviewers of this article have no conflicts of interest.

Received for publication Dec 28, 2020; revisions received Dec 28, 2020; accepted for publication Dec 30, 2020; available ahead of print Jan 1, 2021.

Address for reprints: Danny Ramzy, MD, PhD, Department of Cardiac Surgery, Smidt Heart Institute, Cedars-Sinai Medical Center, 127 S San Vicente Blvd, Suite A3105, Los Angeles, CA 90048 (E-mail: danny.ramzy@cshs.org).

JTCVS Open 2021;6:116-7

2666-2736

Copyright $(2020$ The Authors. Published by Elsevier Inc. on behalf of The American Association for Thoracic Surgery. This is an open access article under the CC BY-NCND license (http://creativecommons.org/licenses/by-nc-nd/4.0/).

https://doi.org/10.1016/j.xjon.2020.12.018
Check for updates

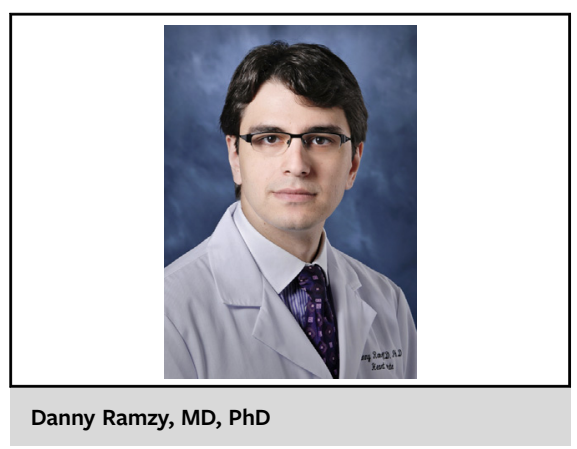

CENTRAL MESSAGE

Direct cellular reprogramming is

a promising strategy in regener-

ative medicine but will require

further validation.

by introducing a specific set of transcription factors (Oct3/4, Sox2, c-Myc, and Klf4). ${ }^{4}$ After achieving this induced pluripotent state, the cells can then be differentiated into specific cell types, including cardiomyocytes. In subsequent work, the intermediate pluripotent state is bypassed by reprogramming fibroblasts directly into cardiomyocytes, using defined transcription factors (Gata4, Mef2c, Tbx5, and Hand2). ${ }^{5,6}$ The translational value of this discovery is further enhanced when similar transdifferentiation is demonstrated using human-derived cells ${ }^{7}$ and when direct reprogramming is demonstrated in vivo. ${ }^{6,8}$ Given that $44 \%$ to $70 \%$ of cells within the heart are noncardiomyocytes, with fibroblasts predominant, ${ }^{9}$ harnessing fibroblasts for conversion is an attractive therapeutic approach. Some limitations must be considered, however.

The efficiency of reprogramming is neither perfect nor complete. In the seminal studies referenced above, for example, some cells closely resembled cardiomyocytes, whereas others were only partially reprogrammed. Yet several factors have been identified that may increase the efficiency of transdifferentiation, including various growth factors, small molecules, and miRNAs. Interestingly, vascular endothelial growth factor (VEGF) has been identified as one such element. ${ }^{10}$ Thus, as Ryan and colleagues point out, VEGF can enhance the therapeutic potential of direct cellular reprogramming not only by increasing conversion rates, but also by rendering an adjuvant effect through angiogenesis.

Another potential barrier to translation is the delivery method. The gene delivery methods discussed above rely primarily on viral vectors, which can have potential 
off-target effects, ectopic reprogramming, and insertional mutagenesis. This final concern of mutagenesis is one of the main factors generating public fear of cellular reprogramming and inhibiting public enthusiasm and funding for such therapy. It does not take much research to know that these fears are pervasive. Fears, whether rational or irrational, of the new COVID-19 mRNA vaccine, with some complaining that it is merely genetic manipulation and refusing to be vaccinated, indicate that we have a long road ahead of us before we can have meaningful success with cellular reprogramming therapies. Therefore, further studies are needed to ensure safe and effective delivery. This includes improved messaging to the overall community to win support for large trials and for broad acceptance and adoption of these therapies. Finally, the mechanisms underlying the functional benefits of cellular reprogramming remain unclear. Although replacement of scar tissue with cardiomyocytes is the suggested mechanism for improved cardiac function, indirect and paracrine effects also may play a role, as they do in stem/progenitor cellbased therapies.

After decades of underachieving in this area, direct transdifferentiation of fibroblasts into cardiomyocytes represents a promising new paradigm in regenerative medicine. Unlike cell-based therapies and subsequent investigations of their secreted paracrine factors, cellular reprogramming is in the relatively nascent stages of development. The mantra of academic medicine has long been "from the bench to the bedside." Researchers, clinicians, and clinicianscientists must collaborate and learn from previous research to move their therapies into clinical trials and applications.
Ryan and colleagues' conclusion that cellular reprogramming therapies are needed and have significant potential to impact morbidity and mortality from CAD is correct. However, future clinical studies will ultimately determine the therapeutic utility of this approach. Finally, we stress that our success will be achieved only if we do a masterful job communicating these findings to both the cardiac surgical community and, more importantly, the public.

\section{References}

1. Porrello ER, Mahmoud AI, Simpson E, Hill JA, Richardson JA, Olson EN, et al. Transient regenerative potential of the neonatal mouse heart. Science. 2011;331: $1078-80$.

2. Bergmann O, Bhardwaj RD, Bernard S, Zdunek S, Barnabe-Heider F, Walsh S, et al. Evidence for cardiomyocyte renewal in humans. Science. 2009;324:98-102

3. Ryan CT, Patel V, Rosengart TK. Clinical potential of angiogenic therapy and cellular reprogramming. J Thorac Cardiovasc Surg Open. 2021;6:108-15.

4. Takahashi K, Yamanaka S. Induction of pluripotent stem cells from mouse embryonic and adult fibroblast cultures by defined factors. Cell. 2006;126:663-76.

5. Ieda M, Fu JD, Delgado-Olguin P, Vedantham V, Hayashi Y, Bruneau BG, et al. Direct reprogramming of fibroblasts into functional cardiomyocytes by defined factors. Cell. 2010;142:375-86.

6. Song K, Nam YJ, Luo X, Qi X, Tan W, Huang GN, et al. Heart repair by reprogramming non-myocytes with cardiac transcription factors. Nature. 2012;485: 599-604.

7. Wada R, Muraoka N, Inagawa K, Yamakawa H, Miyamoto K, Sadahiro T, et al. Induction of human cardiomyocyte-like cells from fibroblasts by defined factors. Proc Natl Acad Sci U S A. 2013;110:12667-72.

8. Qian L, Huang Y, Spencer CI, Foley A, Vedantham V, Liu L, et al. In vivo reprogramming of murine cardiac fibroblasts into induced cardiomyocytes. Nature. 2012;485:593-8.

9. Snider P, Standley KN, Wang J, Azhar M, Doetschman T, Conway SJ. Origin of cardiac fibroblasts and the role of periostin. Circ Res. 2009;105:934-47.

10. Yamakawa H, Muraoka N, Miyamoto K, Sadahiro T, Isomi M, Haginiwa S, et al. Fibroblast growth factors and vascular endothelial growth factor promote cardiac reprogramming under defined conditions. Stem Cell Rep. 2015;5: $1128-42$. 